\title{
Antidepressants and Suicide Attempts in Children
}

WHAT'S KNOWN ON THIS SUBJECT: Warnings of increased risk for suicidality have been placed on antidepressant medications. Because antidepressants differ in their characteristics, including effects on neurotransmitters, it is possible that the risk of suicidal behavior for individual medications may differ.

WHAT THIS STUDY ADDS: In a cohort of 36842 children with review of medical records for confirmation of suicide attempts, we found no difference for risk of suicide attempts among individual medications compared with fluoxetine, the currently recommended antidepressant for treatment of depression.
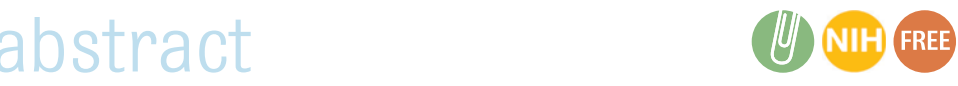

OBJECTIVES: Recent data showing possible increased risk for suicidal behavior among children and adolescents treated with selective serotonin reuptake inhibitors (SSRIs) and serotonin-norepinephrine reuptake inhibitors (SNRIs) antidepressants have created significant concern among patients, families, and providers, including concerns about the risk of individual antidepressants. This study was designed to compare the risk for medically treated suicide attempts among new users of sertraline, paroxetine, citalopram, escitalopram, and venlafaxine to risk for new users of fluoxetine.

METHODS: A retrospective cohort study included 36842 children aged 6 to 18 years enrolled in Tennessee Medicaid between 1995 and 2006 who were new users of 1 of the antidepressant medications of interest (defined as filling no prescriptions for antidepressants in the preceding 365 days). Medically treated suicide attempts were identified from Medicaid files and vital records and confirmed with medical record review.

RESULTS: Four hundred nineteen cohort members had a medically treated suicide attempt with explicit or inferred attempt to die confirmed through medical record review, including 4 who completed suicide. The rate of confirmed suicide attempts for the study drugs ranged from 24.0 per 1000 person-years to 29.1 per 1000 person-years. The adjusted rate of suicide attempts did not differ significantly among current users of SSRI and SNRI antidepressants compared with current users of fluoxetine. Users of multiple antidepressants concomitantly had increased risk for suicide attempt.

CONCLUSIONS: In this population-based study of children recently initiating an antidepressant, there was no evidence that risk of suicide attempts differed for commonly prescribed SSRI and SNRI antidepressants. Pediatrics 2014;133:204-210
AUTHORS: William 0. Cooper, MD, MPH, a,b $\mathrm{S}$. Todd Callahan, MD, MPH, ${ }^{a}$ Ayumi Shintani, PhD, ${ }^{c}$ D. Catherine Fuchs, MD, ${ }^{d}$

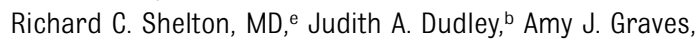
$\mathrm{SM}, \mathrm{MPH}^{\mathrm{c}}$ and Wayne A. Ray, $\mathrm{PhD}^{\mathrm{b}}$

Departments of ${ }^{a}$ Pediatrics, ${ }^{b}$ Health Policy, ${ }^{c}$ Biostatistics, and aPsychiatry, Vanderbilt University School of Medicine, Nashville, Tennessee; and ${ }^{\text {DDepartment }}$ of Psychiatry, University of Alabama Birmingham, Birmingham, Alabama

KEY WORDS

antidepressants, suicide, depression

\section{ABBREVIATIONS}

FDA-Food and Drug Administration

MDD-major depressive disorder

SNRI—serotonin-norepinephrine reuptake inhibitor

SSRI—selective serotonin reuptake inhibitor

Dr Cooper conceptualized and designed the study, oversaw all aspects of study performance, and drafted the initial manuscript; Dr Callahan participated in design of the study, supervised the data collection, served as an adjudicator for suicide cases, and reviewed and revised the manuscript; $D r$ Shintani carried out the initial analyses and reviewed and revised the manuscript; Drs Fuchs and Shelton participated in design of the study, served as an adjudicator for suicide cases, and reviewed and revised the manuscript; Ms Dudley participated in design of the study, carried out the data acquisition, and critically reviewed and revised the manuscript; Ms Graves participated in the analyses and reviewed and revised the manuscript; Dr Ray participated in design of the study, provided input into the analyses and interpretation of data, and reviewed and revised the manuscript; and all authors approved the final manuscript as submitted.

www.pediatrics.org/cgi/doi/10.1542/peds.2013-0923

doi:10.1542/peds.2013-0923

Accepted for publication 0ct 30, 2013

Address correspondence to William 0. Cooper, MD, MPH, Suite 313 Oxford House, 1313 21st Ave South, Nashville, TN 37232-4313.

E-mail: william.cooper@vanderbilt.edu

PEDIATRICS (ISSN Numbers: Print, 0031-4005; Online, 1098-4275) Copyright (C) 2014 by the American Academy of Pediatrics FINANCIAL DISCLOSURE: The authors have indicated they have no financial relationships relevant to this article to disclose.

FUNDING: All phases of this study were supported by a grant from the National Institute of Mental Health (grant MH 079903) Funded by the National Institutes of Health $(\mathrm{NIH})$.

POTENTIAL CONFLICT OF INTEREST: Dr Shelton has consulted for Eli Lilly and Pfizer and has received support from Eli Lilly, Euthymics Bioscience, Forst Pharmaceuticals, and Pfizer. The other authors have indicated they have no potential conflicts of interest to disclose. 
Major depressive disorder (MDD) is a common condition in children and adolescents ${ }^{1,2}$ and has significant negative impacts on children's development, functioning, and risk for suicide. ${ }^{2-5}$ Antidepressant medications are often used to treat childhood depression, but concerns about possible increased risk for suicidal behavior among children and adolescents treated with antidepressants led to warnings from the US Food and Drug Administration (FDA) and the UK Medicine Healthcare Products Regulatory Agency.6-9

Regulatory warnings for suicide risk in children and adolescents treated with antidepressants do not distinguish individual antidepressants, but data from clinical trials suggested that the risk might differ for individual drugs, particularly for newer selective serotonin reuptake inhibitors (SSRIs) and serotoninnorepinephrine reuptake inhibitors (SNRIs).9,10 Individual drug characteristics, such as neurotransmitter selectivity or elimination half-life, could differentially influence the neurobiological processes of suicide, including impulsivity and hopelessness. ${ }^{11-13}$ For example, the SNRI drug venlafaxine has both serotonergic and noradrenergic effects, whereas SSRIs have more selective serotonergic effects. ${ }^{12,13}$ Understanding more about the potential risks for suicides related to individual antidepressants would provide important information for patients, families, and providers to guide treatment decisions.

Thus, we conducted an observational study with a cohort of children who were new users of individual SSRI and SNRI antidepressants and conducted medical record review for all suicide attempts identified from Medicaid claims and vital records. We compared the risk for medically treated suicide attempts for sertraline, paroxetine, citalopram, escitalopram, and venlafaxine to fluoxetine, the currently FDA-recommended antidepressant for treatment of depression in children and adolescents.

\section{METHODS}

\section{Design and Data Sources}

We assembled a retrospective cohort of children, aged 6 to 18 years, enrolled in Tennessee Medicaid from 1995 to 2006. Tennessee Medicaid files linked with state death certificates and statewide hospital discharge data provided an efficient source for identifying the cohort, determining periods of probable exposure to medications, and ascertaining end points. The Medicaid data included enrollment records, outpatient and inpatient claims, and records of filled prescriptions (including dispensing date, drug name, dose, quantity, and days of supply), which have been shown to have concordance of $\geq 90 \%$ with patient self-report of medication use. ${ }^{14-17}$

\section{Study Population}

To assemble the cohort, we identified new users of fluoxetine, sertraline, paroxetine, citalopram, escitalopram, or venlafaxine, defined as those who filled no prescriptions for an antidepressant during the preceding 365 days. Restricting the cohort to new users allowed the study to identify end points that may occur early in treatment. ${ }^{18}$ Fluvoxamine, another SSRI antidepressant marketed during the study period, was not included because it is primarily used for different indications, and there was limited use in the Tennessee Medicaid population ( $n=261$ new users). Cohort members had continuous Medicaid enrollment during the 365 days before the first prescription and had no evidence of major mental illness other than MDD (defined as filling prescriptions for lithium, antipsychotics, mood stabilizing anticonvulsants in the absence of a seizure diagnosis, or claims with a diagnosis of bipolar disorder, psychosis, or schizophrenia including $\geq 1$ inpatient or 2 outpatient claims). We also excluded the small number of children with life-threatening illness in the 365 days before the first prescription $(<1 \%)$ because these children may have an atypical risk of suicidality. Children were followed until the earliest of the last study day, their 19th birthday, death, loss of enrollment of $\geq 7$ days (typically $\leq 10 \%$ ), development of an exclusion illness, no filling of antidepressant prescriptions for 180 consecutive days, or a suicide attempt. Once children left the cohort, they were allowed to reenter if they met eligibility requirements again.

\section{Antidepressant Use}

Every person-day during study followup was classified according to use of 1 of the study antidepressants. Current use of an antidepressant was defined as the period between the prescription start date and the end of the days of supply (including up to a 7-day carryover period from previous prescriptions). Follow-up days with current use of $\geq 2$ antidepressants concomitantly were included in a separate exposure category for multiple antidepressants. We also recorded person time after current use ended but focused on current use for this study to capture the potential acute effects of study medications on the study end point.

\section{Study End Points}

Medically treated suicide attempts were defined as self-harm associated with a stated or inferred intent to die. Suicide deaths were defined as selfharm associated with death. Potential end points were identified from computerized records for inpatient, emergency department, and outpatient encounters and included International Classification of Diseases (9th and 10th revisions) codes used in previous population-based studies of suicide epidemiology. ${ }^{19,20}$ Because we planned to review medical records for all end points, the algorithm to identify cases 
was intentionally broad and included injuries that might reasonably be related to self-harm (eg, all claims for falls from a height, gunshot wounds), as we have previously described. ${ }^{21}$ Medical records, including emergency medical services reports, emergency department records, mental health evaluations, as well as records from medical and psychiatric hospitalizations were reviewed to confirm that the attempt was related to self-harm with an explicit or inferred intent to die, by using the Columbia Suicidality Classification Scale.22 For all deaths in the cohort, we reviewed autopsy and toxicology reports. The principal investigator (WOC) and a second investigator (STC) reviewed all cases (agreement of $100 \%$ on case status for a $10 \%$ random sample of cases). Cases the primary adjudicators considered to be equivocal (8\%) were reviewed by a larger group, which included the 2 adjudicators and a child (DCF) and adult (RCS) psychiatrist. of the equivocal cases, the larger group was able to determine outcome status for all but $2.4 \%$.

We identified 1480 episodes of potential suicidal behavior from medical claims data and vital records. Of these, 1285 (86.8\%) had records available for adjudication. In cases in which we were unable to obtain medical records or had insufficient information for adjudication (13.2\% of cases), we determined the case status using a computer case definition, derived from cases with completed adjudication, which had a positive predictive value of $85 \%$. Using these methods, we identified 419 end points, including 415 confirmed attempts and 4 completed suicides.

\section{Analysis}

The analysis estimated the relative risks for current use of sertraline, paroxetine, citalopram, escitalopram, and venlafaxine compared with current use of fluoxetine by using generalized estimating equation regression models with Poisson distribution.23,24 These regression models were weighted by the inverse of the probability of treatment to adequately adjust for potential confounding. The Poisson distribution was used as it is suitable for rare events. ${ }^{23}$ Generalized estimating equation models accounted for dependency among repeated hospitalizations. ${ }^{24}$ Propensity scores were computed as the probability of being in each antidepressant category on the first day of study follow-up. ${ }^{25}$ Baseline variables used in creating the propensity score included sociodemographic characteristics (age, gender, race, ethnicity, urban/rural residence, enrollment because of disability, and calendar year and month at study entry) as well as information on medical care encounters in the previous 365 days consistent with psychiatric disorders, asthma, diabetes, seizures, and thyroid disease, as well as medications used to treat these conditions, visits associated with psychiatric diagnoses in inpatient, outpatient, and emergency department settings, and previous suicide attempts. Inverse probability of treatment weighting based on the propensity scores was used to adjust for differences between the antidepressant exposure groups. ${ }^{26}$ Inverse propensity score weights were trimmed at the 99th percentile to prevent bias from including overly influential points. ${ }^{27}$ To demonstrate the adequacy of the propensity score models in achieving covariate balance, we compared the mean of each propensity score before and after inverse probability of treatment weighting (Supplemental Table 3).

In our primary models, we adjusted for medication-specific propensity score and several time-dependent covariates (age, calendar year and month, mental health diagnoses, and use of psychotropic medications). Additional analyses were stratified by gender, age, and presence of a suicide attempt before study entry. We performed all analyses with SAS 9.1 (SAS Institute, Cary, NC) and R 3.0 (www.r-project.org).

\section{Human Subjects Protection}

The study was approved by the institutional review board at Vanderbilt University, the Tennessee Department of Health, and the TennCare Bureau.

\section{RESULTS}

\section{Study Population}

The cohort included 36842 children who were new users of study antidepressants during the study period (Table 1). The mean age of new users of the drugs of interest was 14.0 years; $59.3 \%$ were female; $15.9 \%$ were African American; and $30.2 \%$ resided in urban settings. In the year preceding cohort entry, just less than one-half (47.4\%) of the cohort members had a diagnosis of MDD, and approximately one-fourth had a diagnosis of attention-deficit/ hyperactivity disorder. Other common psychiatric conditions among cohort members included conduct disorder and anxiety. Approximately $10 \%$ of the cohort members had asthma, $2 \%$ to $3 \%$ had seizures, and $1.6 \%$ had diabetes. Before entering the cohort, $10.0 \%$ had been hospitalized in a psychiatric facility, and $2.9 \%$ had a medically treated suicide attempt.

The study drug exposure groups were comparable in terms of demographic characteristics, although venlafaxine and escitalopram users were somewhat older than fluoxetine users, and a smaller proportion of venlafaxine users lived in urban settings (Table 1). The exposure groups were also comparable in the proportion with depression and attention-deficit/hyperactivity disorder diagnoses, but a smaller proportion of fluoxetine users had anxiety diagnoses compared with the other study drugs. A higher proportion of venlafaxine users also filled prescriptions 
TABLE 1 Demographic and Clinical Characteristics at Baseline of Children and Adolescents in Tennessee Medicaid Who Were New Users of Antidepressant Medications During the Study Period

\begin{tabular}{|c|c|c|c|c|c|c|c|}
\hline Characteristic & Fluoxetine & Sertraline & Paroxetine & Citalopram & Escitalopram & Venlafaxine & Multiple SSRIS \\
\hline$N$ persons & 7967 & 10495 & 8769 & 3302 & 2791 & 1684 & 1834 \\
\hline Calendar year of study entry, t0 & 2001.0 & 2002.5 & 2001.0 & 2002.7 & 2004.1 & 2002.3 & 2001.5 \\
\hline \multicolumn{8}{|l|}{ Demographics at study entry } \\
\hline Age, y, mean (SD) & $13.8(3.2)$ & $13.7(3.3)$ & $14.0(3.2)$ & $14.1(3.3)$ & $14.9(2.8)$ & $14.8(3.1)$ & $14.7(2.8)$ \\
\hline Female gender, \% & 60.1 & 57.5 & 57.5 & 60.0 & 64.4 & 62.2 & 62.9 \\
\hline African American race, \% & 15.8 & 16.8 & 15.5 & 16.6 & 16.0 & 10.0 & 17.2 \\
\hline Hispanic ethnicity, \% & 0.8 & 1.0 & 0.7 & 0.7 & 0.9 & 0.6 & 0.7 \\
\hline Urban residence, \% & 32.5 & 31.4 & 30.9 & 26.1 & 27.5 & 20.0 & 30.9 \\
\hline Disability, \% & 13.8 & 12.1 & 12.6 & 11.8 & 11.3 & 8.6 & 11.2 \\
\hline \multicolumn{8}{|l|}{ Psychiatric conditions, past year, \% } \\
\hline MDD & 48.2 & 46.0 & 46.2 & 50.1 & 45.8 & 44.3 & 58.5 \\
\hline ADHD & 24.8 & 27.4 & 25.8 & 26.6 & 19.8 & 21.4 & 19.8 \\
\hline Conduct disorder & 18.0 & 17.9 & 18.5 & 18.3 & 13.8 & 14.0 & 21.2 \\
\hline Anxiety & 14.0 & 19.6 & 23.2 & 17.3 & 17.9 & 20.1 & 19.4 \\
\hline Alcohol/drug dependence and abuse & 7.3 & 7.3 & 7.9 & 9.2 & 10.0 & 10.1 & 14.6 \\
\hline Adjustment reaction & 5.2 & 3.9 & 5.8 & 4.7 & 3.0 & 3.4 & 8.7 \\
\hline Mental retardation & 4.4 & 4.3 & 4.0 & 4.3 & 3.3 & 2.1 & 2.5 \\
\hline Other psychiatric & 4.4 & 5.5 & 4.8 & 5.4 & 4.2 & 3.6 & 9.1 \\
\hline Eating disorder & 2.3 & 1.9 & 2.3 & 2.2 & 2.1 & 1.9 & 2.8 \\
\hline \multicolumn{8}{|l|}{ Psychiatric meds, past year, \% } \\
\hline ADHD medications ${ }^{a}$ & 14.6 & 13.8 & 11.7 & 11.7 & 11.6 & 9.1 & 5.6 \\
\hline Other ADHD medications ${ }^{b}$ & 4.7 & 4.2 & 4.1 & 4.0 & 2.7 & 2.4 & 2.9 \\
\hline Anxiolytics ${ }^{c}$ & 3.4 & 3.7 & 4.4 & 3.9 & 5.2 & 6.5 & 3.9 \\
\hline \multicolumn{8}{|l|}{ Other conditions, past year, $\%$} \\
\hline Asthma & 9.1 & 10.4 & 9.5 & 9.0 & 9.7 & 9.7 & 10.8 \\
\hline Pain & 3.3 & 4.3 & 4.5 & 3.7 & 5.6 & 6.4 & 1.9 \\
\hline Seizures & 3.1 & 2.7 & 2.6 & 3.4 & 2.8 & 2.3 & 2.0 \\
\hline Headache & 2.5 & 3.6 & 3.5 & 3.4 & 4.5 & 4.0 & 2.4 \\
\hline Fatigue and malaise & 2.1 & 2.2 & 2.6 & 2.5 & 3.5 & 4.1 & 1.6 \\
\hline Diabetes & 1.8 & 1.3 & 1.5 & 1.8 & 2.1 & 1.8 & 1.3 \\
\hline Premenstrual syndrome & 1.7 & 0.8 & 0.8 & 0.6 & 0.9 & 0.7 & 0.3 \\
\hline Thyroid disease & 1.2 & 1.2 & 1.2 & 0.9 & 1.1 & 1.5 & 0.7 \\
\hline Enuresis & 0.4 & 0.4 & 0.4 & 0.2 & 0.2 & 0.2 & 0.4 \\
\hline Tobacco use disorder & 0.3 & 0.5 & 0.5 & 0.2 & 0.8 & 0.4 & 0.1 \\
\hline Insomnia & 0.1 & 0.1 & 0.1 & 0.0 & 0.1 & 0.1 & 0.3 \\
\hline \multicolumn{8}{|l|}{ Use of health care, past $y, \%$} \\
\hline Psychiatric hospitalization & 11.9 & 7.3 & 10.2 & 10.6 & 8.3 & 6.0 & 22.3 \\
\hline Nonpsychiatric hospitalization & 11.4 & 11.5 & 10.9 & 11.6 & 13.3 & 12.6 & 13.7 \\
\hline Previous suicide attempt & 3.2 & 2.4 & 2.3 & 3.6 & 2.9 & 2.0 & 6.7 \\
\hline Treated injury & 27.3 & 27.6 & 28.8 & 29.5 & 26.9 & 29.2 & 34.3 \\
\hline
\end{tabular}

ADHD, attention-deficit/hyperactivity disorder.

a Medications included amphetamines, methylphenidate, and atomoxetine.

b Included clonidine and guanfacine.

c Included benzodiazepines, chloral hydrate, ramelteon, and buspirone.

for anxiolytics in the year preceding cohort entry. Venlafaxine users were also somewhat less likely to have a psychiatric hospitalization than users of other antidepressants, but approximately the same proportion had nonpsychiatric hospitalizations. The groups were comparable in terms of the proportion with previous suicide attempts (2.0\%-3.6\%). Cohort members who used multiple antidepressants concomitantly had higher rates of previous psychiatric hospitalizations and more frequent suicide attempts before study entry than fluoxetine users.

\section{Study End Points}

Four hundred nineteen cohort members had a medically treated suicide attempt with explicit or inferred attempt to die confirmed through medical record review, including 4 cohort members who completed suicide. Cohort members with medically treated suicide attempts had a mean age of 15.4 years at the time of the attempt. The rate of confirmed suicide attempts for current users of the study drugs ranged from 24.0 per 1000 person-years to 29.1 per 1000 person-years (Table 2). For current users of sertraline, paroxetine, citalopram, escitalopram, and venlafaxine, the adjusted rate of suicide attempts did not differ significantly from that for current users of fluoxetine (Table 2). Children who used multiple antidepressants 
concomitantly had higher risk for medically treated suicide attempts (adjusted relative risk 1.70, 95\% confidence interval 1.10-2.62) than did fluoxetine users.

\section{Alternative Analyses}

We performed several alternative analyses to test the robustness of study findings (Fig 1). We performed analyses stratified by gender and found no statistically significant difference in the risk for medically treated suicide attempts for either gender for any of the study drugs. In other analyses, we compared the risk among the individual antidepressants when we restricted the cohort to children of age 13 to 18 years and when we restricted the cohort to children who had no history of a suicide attempt in the year preceding cohort entry. Finally, we conducted an analysis restricting the cohort to the final 6 years of the study period to assess the effect of temporal changes in antidepressant prescribing. In these additional analyses, we found no statistically significant differences when current users of the study drugs were compared with current users of fluoxetine (Fig 1).

\section{DISCUSSION}

In this large cohort of 36842 children who were new users of SSRI and SNRI antidepressant medications, we found no evidence that the use of paroxetine, sertraline, citalopram, escitalopram, or venlafaxine was associated with a greater risk of medically treated suicide attempts than fluoxetine. In analyses stratified by gender, older age, absence of a suicide attempt before initiating antidepressants, and data from the most recent years of the study period, there was also no evidence of increased risk for any of the study drugs relative to fluoxetine.

This study addresses many limitations of previous research in this area. The study included a large cohort with racial, ethnic, and sociodemographic diversity, allowing for comparisons of outcomes among individual drugs rather than classes of drugs. Restricting the cohort to adolescents who had not used antidepressants in the previous year (ie, new users) allowed the study to avoid underascertainment of outcomes occurring early in treatment and the inability to control for risk factors for suicide that may be altered by study drugs. ${ }^{18}$ We performed medical record review for all potential suicide attempts and applied a validated algorithm for suicidality.22 Applying these rigorous definitions, the rate of medically treated suicide attempts for SSRI and SNRI users (24-29/1000 person years) was comparable to that

TABLE 2 Risk for Suicide Attempts Among Children Who Were Current Users of Individual Antidepressants Compared With Children and Adolescents Who Were Current Users of Fluoxetine

\begin{tabular}{lcccc}
\hline & $\begin{array}{c}\text { Person-Years of } \\
\text { Current Use }\end{array}$ & Events & $\begin{array}{c}\text { Rate/1000 } \\
\text { Person-Years }\end{array}$ & $\begin{array}{c}\text { Adjusted RR (95\% } \\
\text { Confidence Interval) }\end{array}$ \\
\hline Fluoxetine & 3070 & 76 & 24.8 & 1 \\
Sertraline & 4208 & 104 & 24.7 & $0.97(0.66-1.45)$ \\
Paroxetine & 2956 & 71 & 24.0 & $0.80(0.52-1.21)$ \\
Citalopram & 1271 & 37 & 29.1 & $0.92(0.57-1.50)$ \\
Escitalopram & 1066 & 27 & 25.3 & $0.80(0.45-1.43)$ \\
Venlafaxine & 721 & 21 & 29.1 & $0.80(0.43-1.51)$ \\
Multiple SSRls only & 1354 & 83 & 61.3 & $1.70(1.10-2.62)$ \\
\hline
\end{tabular}

a Relative risks (RRs) were estimated with generalized estimate equation regression models with Poisson distribution and were weighted by the inverse of a propensity score for each medication, age, calendar year, psychiatric conditions (use of psychotropic medications and diagnoses for serious psychiatric disorders), and utilization of health care (psychiatric and medical hospitalizations, and unintentional injuries). Inverse propensity score weights were trimmed at the 99th percentile due to a few large, influential weights.

described by Schneeweiss et al using data from Canada and in the US FDA's descriptions of suicidality and antidepressants (20-40/1000). ${ }^{28,29}$ We controlled for several important covariates, including measures of use of mental health services, by calculating a propensity score at baseline for all cohort members and by using inverse propensity score weighting in multivariate models.

Previous analyses of clinical trials data suggested that there may be differences in the risk of suicidality among antidepressants, including particular evidence of increased risk for venlafaxine. ${ }^{8}$ We found no evidence of increased risk for venlafaxine or any of the other drugs studied, although the point estimate for venlafaxine was higher than that for fluoxetine. Our study findings suggest that we can rule out potential absolute excess risk of 13 per 1000 person years for venlafaxine compared with fluoxetine. Given the purported role of increased levels of anxiety in suicide risk related to antidepressants, 6,7 it is interesting to note that a higher proportion of venlafaxine users had comorbid anxiety diagnoses, and a greater proportion filled prescriptions for anxiolytics in the year preceding study entry. We found increased risk for suicidal behavior for study participants who had overlapping use of multiple antidepressants, which likely reflects the increased severity of depression for these individuals rather than a drug effect. ${ }^{30}$

The study has some limitations. By focusing on suicide attempts resulting in death or medical treatment, we potentially missed less serious attempts or suicidal thoughts. We chose to focus on treated attempts and completed suicides because we considered these to be more serious given that they resulted in greater use of medical care and represented a greater likelihood of medical or psychiatric morbidity than 


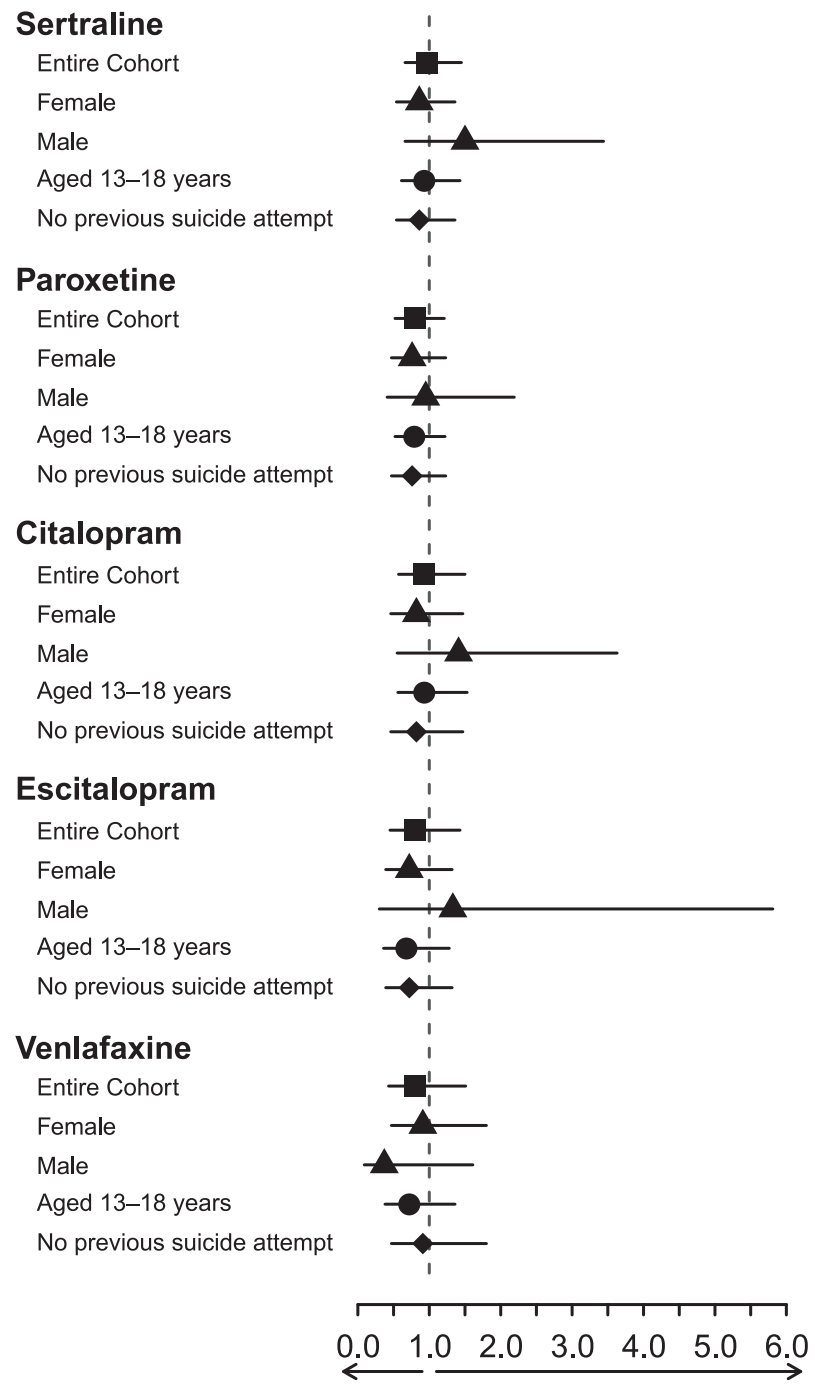

Better than Fluoxetine Worse than Fluoxetine

\section{Relative Risk (95\% Confidence Interval)}

\section{FIGURE 1}

Alternative analyses, risk for suicide attempt among children and adolescents who were current users of individual antidepressants compared with children and adolescents who were current users of fluoxetine.

nontreated events. ${ }^{4}$ It is also possible that use of individual study drugs was guided by a child's mental illness severity, resulting in confounding by indication. We included several measures of mental health services use in propensity scores and time varying covariates in our analyses to mitigate the role of such confounding. We were unable to study the specific risk for completed suicides because of the small number of these events in our cohort. Finally, it is possible that children and adolescents in the Medicaid program may differ from other children because of increased exposure to poverty and other life stressors. Although this may be true, Medicaid provides insurance for a large proportion of children in Tennessee and the United States. ${ }^{31}$ These children are typically underrepresented in clinical trials and thus represent an important population for study. The study was not designed to identify risk for suicide attempts for antidepressant users compared with nonusers. Analyses of clinical trials data by the FDA and others suggested potential differences among individual antidepressants, 7,32 although power to detect differences among individual medications was limited in the FDA's analyses. Thus, we chose to focus the current study on the important question of individual medication risk. Additional work is needed to further characterize the relationship between antidepressant use and suicidal behaviors.

\section{CONCLUSIONS}

We found no evidence of increased risk for SSRI or SNRI antidepressants compared with fluoxetine for new users of antidepressants in the Tennessee Medicaid population. Use of any of the individual drugs studied would not be expected to increase risk for serious suicide attempts compared with the others.

\section{ACKNOWLEDGMENTS}

We thank the TennCare Bureau and the Tennessee Department of Health, which provided data needed to conduct the study. We thank the following individuals who provided assistance with the study: Patricia A. Gideon, Michelle DeRanieri, Leanne Balmer, Shannon D. Stratton, Lynne Caples, Tracy Crowley, and Ning Chen. 


\section{REFERENCES}

1. Kessler RC, Berglund P, Demler 0, Jin R, Merikangas KR, Walters EE. Lifetime prevalence and age-of-onset distributions of DSM-IV disorders in the National Comorbidity Survey Replication [published correction appears in Arch Gen Psychiatry. 2005;62(7):768]. Arch Gen Psychiatry. 2005; 62(6):593-602

2. Birmaher B, Brent D, Bernet W, et al; AACAP Work Group on Quality Issues. Practice parameter for the assessment and treatment of children and adolescents with depressive disorders. J Am Acad Child Adolesc Psychiatry. 2007;46(11):1503-1526

3. Bridge JA, Goldstein TR, Brent DA. Adolescent suicide and suicidal behavior. J Child Psychol Psychiatry. 2006;47(3-4):372-394

4. Hawton K, Saunders KE, O'Connor RC. Selfharm and suicide in adolescents. Lancet. 2012;379(9834):2373-2382

5. Cash SJ, Bridge JA. Epidemiology of youth suicide and suicidal behavior. Curr Opin Pediatr. 2009;21(5):613-619

6. Leslie LK, Newman TB, Chesney PJ, Perrin $\mathrm{JM}$. The Food and Drug Administration's deliberations on antidepressant use in pediatric patients. Pediatrics. 2005;116(1): 195-204

7. Mosholder AD. Suicidality in pediatric clinical trials with paroxetine and other antidepressants. Updated February 18, 2004. Available at: http://psychrights.org/ research/Digest/AntiDepressants/Mosholder/ MosholderReport.pdf. Accessed March 15, 2013

8. Hammad TA, Laughren T, Racoosin J. Suicidality in pediatric patients treated with antidepressant drugs. Arch Gen Psychiatry. 2006;63(3):332-339

9. Hetrick SE, McKenzie JE, Cox GR, Simmons MB, Merry SN. Newer generation antidepressants for depressive disorders in children and adolescents. Cochrane Database Syst Rev. 2012;11:CD004851

10. Gibbons RD, Brown CH, Hur K, Davis J, Mann JJ. Suicidal thoughts and behavior with antidepressant treatment: reanalysis of the randomized placebo-controlled studies of fluoxetine and venlafaxine. Arch Gen Psychiatry. 2012;69(6):580-587

11. Mann JJ. Neurobiology of suicidal behaviour. Nat Rev Neurosci. 2003;4(10):819-828

12. Ryan ND. Treatment of depression in children and adolescents. Lancet. 2005;366 (9489):933-940

13. Shelton RC, Lester N. Selective serotonin reuptake inhibitors and new anti-depressants. In: Stein DJ, Kupfer DJ, Schatzburg AF, eds. The American Psychiatric Publishing Textbook of Mood Disorders. Arlington, VA: American Psychiatric Publishing; 2006:263-281

14. Johnson RE, Vollmer WM. Comparing sources of drug data about the elderly. $J$ Am Geriatr Soc. 1991;39(11):1079-1084

15. Ray WA, Griffin MR. Use of Medicaid data for pharmacoepidemiology. Am J Epidemiol. 1989;129(4):837-849

16. Ray WA. Population-based studies of adverse drug effects. N Engl J Med. 2003;349 (17):1592-1594

17. West SL, Savitz DA, Koch G, Strom BL, Guess HA, Hartzema A. Recall accuracy for prescription medications: self-report compared with database information. Am J Epidemiol. 1995;142(10):1103-1112

18. Ray WA. Evaluating medication effects outside of clinical trials: new-user designs. Am J Epidemiol. 2003;158(9):915-920

19. Olfson M, Gameroff MJ, Marcus SC, Greenberg $T$, Shaffer $D$. National trends in hospitalization of youth with intentional selfinflicted injuries. Am J Psychiatry. 2005; 162(7):1328-1335

20. Doshi A, Boudreaux ED, Wang N, Pelletier AJ, Camargo CA Jr. National study of US emergency department visits for attempted suicide and self-inflicted injury, 19972001. Ann Emerg Med. 2005;46(4):369-375

21. Callahan ST, Fuchs DC, Shelton RC, et al. Identifying suicidal behavior among adolescents using administrative claims data. Pharmacoepidemiol Drug Saf. 2013;22(7): 769-775

22. Posner K, Oquendo MA, Gould M, Stanley B, Davies M. Columbia Classification Algo- rithm of Suicide Assessment (C-CASA): classification of suicidal events in the FDA's pediatric suicidal risk analysis of antidepressants. Am J Psychiatry. 2007;164 (7): 1035-1043

23. Zou G. A modified poisson regression approach to prospective studies with binary data. Am J Epidemiol. 2004;159(7): 702-706

24. Zeger SL, Liang KY, Albert PS. Models for Iongitudinal data: a generalized estimating equation approach. Biometrics. 1988;44(4): 1049-1060

25. Rosenbaum PR, Rubin DB. The central role of the propensity score in observational studies for causal effects. Biometrika. 1983; 70(1):41-55

26. Rosenbaum PR. Model-based direct adjustment. J Am Stat Assoc. 1987;82(398): 387-394

27. Lee BK, Lessler J, Stuart EA. Weight trimming and propensity score weighting. PLoS ONE. 2011;6(3):e18174

28. Schneeweiss S, Patrick AR, Solomon DH, et al. Comparative safety of antidepressant agents for children and adolescents regarding suicidal acts. Pediatrics. 2010;125 (5):876-888

29. Food and Drug Administration. Labeling change request letter for antidepressant medications. Available at: www.fda.gov/Drugs/DrugSafety/ InformationbyDrugClass/ucm096352.htm. Published 2005. Accessed August 2, 2013

30. Fontanella CA, Bridge JA, Campo JV. Psychotropic medication changes, polypharmacy, and the risk of early readmission in suicidal adolescent inpatients. Ann Pharmacother. 2009;43(12):1939-1947

31. Mirvis DM, Chang CF. Tennessee's option to expand Medicaid coverage: what are the issues? Tenn Med. 2012;105(10): 27-31

32. Hammad T. Results of the analysis of suicidality in pediatric trials of new antidepressants. Joint Meeting of the Psychopharmacologic Drugs Advisory Committee and the FDA Pediatric Advisory Committee; September 13, 2004; Bethesda, MD 